\title{
Pyrolytic carbon resonators for micromechanical thermal analysis
}

Long Quang Nguyen (10 ${ }^{1,2}$, Peter Emil Larsen ${ }^{1,3}$, Tom Larsen ${ }^{4}$, Sanjukta Bose Goswami ${ }^{1,3}$, Luis Guillermo Villanueva (1) ${ }^{4}$, Anja Boisen ${ }^{1,3}$ and Stephan Sylvest Keller ${ }^{1,2}$

\begin{abstract}
Thermal analysis is essential for the characterization of polymers and drugs. However, the currently established methods require a large amount of sample. Here, we present pyrolytic carbon resonators as promising tools for micromechanical thermal analysis (MTA) of nanograms of polymers. Doubly clamped pre-stressed beams with a resonance frequency of $233 \pm 4 \mathrm{kHz}$ and a quality factor ( $\mathrm{Q}$ factor) of $800 \pm 200$ were fabricated. Optimization of the electrical conductivity of the pyrolytic carbon allowed us to explore resistive heating for integrated temperature control. MTA was achieved by monitoring the resonance frequency and quality factor of the carbon resonators with and without a deposited sample as a function of temperature. To prove the potential of pyrolytic carbon resonators as thermal analysis tools, the glass transition temperature $\left(T_{g}\right)$ of semicrystalline poly $(L-l a c t i c$ acid) (PLLA) and the melting temperature $\left(T_{\mathrm{m}}\right)$ of poly(caprolactone) (PCL) were determined. The results show that the $T_{\mathrm{g}}$ of PLLA and $T_{\mathrm{m}}$ of PCL are $61.0 \pm 0.8^{\circ} \mathrm{C}$ and $60.0 \pm 1.0^{\circ} \mathrm{C}$, respectively, which are in excellent agreement with the values measured by differential scanning calorimetry (DSC).
\end{abstract}

\section{Introduction}

Thermal analysis refers to a number of different techniques involving temperature control to study the material properties of a sample. These techniques include thermogravimetry (TGA), dynamic mechanical analysis (DMA), differential thermogravimetry (DTG), differential scanning calorimetry (DSC), differential thermal analysis (DTA) and temperature-programmed desorption (TPD). The information obtained through thermal analysis provides an understanding of thermal properties, such as the glass transition temperature $T_{\mathrm{g}}$ or the melting temperature $T_{\mathrm{m}}$, of a variety of materials, such as polymers ${ }^{1,2}$, pharmaceutical compounds ${ }^{3}$ or proteins ${ }^{4}$. However, these techniques typically require a few milligrams of sample, which is not always available or only available at high costs. To address

Correspondence: Long Quang Nguyen (longq@dtu.dk)

${ }^{1}$ DNRF and Villum Fonden Center for Intelligent Drug Delivery and Sensing Using Microcontainers and Nanomechanics, IDUN, Technical University of Denmark, 2800 Kgs Lyngby, Denmark

${ }^{2}$ DTU Nanolab, Technical University of Denmark, 2800 Kgs Lyngby, Denmark Full list of author information is available at the end of the article. this limitation, thermal analysis using microelectromechanical system (MEMS) structures, such as freestanding beams or membranes, has been introduced. The principle of this method is based on tracking the resonance frequency shift and $\mathrm{Q}$ factor of the micromechanical resonators during controlled temperature ramps, followed by correlating the resonant behavior with changes in the thermal properties of the analyzed sample. Resonating sensors, such as quartz microresonators ${ }^{5,6}$, micromechanical cantilevers ${ }^{5,7}$, and micromechanical string resonators ${ }^{8,9}$, have been employed for micromechanical thermal analysis (MTA) of nanogram samples due to the small size of the sensors. The excellent performance of resonating microsensors, specifically the high sensitivity and the short thermal response time, has been explored for various thermal analysis applications ${ }^{10-16}$. Furthermore, micromechanical resonators are suitable for integration with Si-based microelectronics.

To date, in order to use micromechanical resonators for thermal analysis, an external heat source, such as a Peltier element $^{8}$, a metal ceramic heater ${ }^{9}$ or a holder with

\section{(c) The Author(s) 2019}

(c) (i) Open Access This article is licensed under a Creative Commons Attribution 4.0 International License, which permits use, sharing, adaptation, distribution and reproduction cc) in any medium or format, as long as you give appropriate credit to the original author(s) and the source, provide a link to the Creative Commons license, and indicate if changes were made. The images or other third party material in this article are included in the article's Creative Commons license, unless indicated otherwise in a credit line to the material. If material is not included in the article's Creative Commons license and your intended use is not permitted by statutory regulation or exceeds the permitted use, you will need to obtain permission directly from the copyright holder. To view a copy of this license, visit http://creativecommons.org/licenses/by/4.0/. 
temperature control ${ }^{17}$, has been used. This approach results in a relatively complicated setup for controlling the temperature during MTA and a slow thermal response due to the large thermal mass of the measurement system. Therefore, direct integration of heating and temperature control into the resonators would simplify the setup and significantly reduce the time constant during the measurements. In several studies, microheaters have been integrated with quartz crystal microbalance (QCM) sensors for measuring particulate matter ${ }^{18}$ or the viscosity of materials ${ }^{19}$. Furthermore, microheaters have also been integrated with suspended membranes for gas sensing ${ }^{20}$ or doubly clamped beams for calorimetry ${ }^{21}$. To integrate these microheaters, metals, such as $\mathrm{Au}$ or $\mathrm{Pt}$, have been fabricated as patterned resistor structures on the sensors. However, this approach requires more steps in the fabrication process, which is time consuming and expensive.

In the past decade, carbon MEMS (C-MEMS) has been established as a method to fabricate carbon microstructures. Basically, in the C-MEMS process, a photoresist precursor structure is pyrolysed at high temperature in an inert atmosphere and converted into pyrolytic carbon $^{22,23}$. To date, pyrolytic carbon has been successfully applied in electrochemistry. By using pyrolytic carbon as an electrode material, many electrochemical ${ }^{24,25}$ or bionanoelectronics ${ }^{26}$ applications have been developed. Furthermore, 3D pyrolytic carbon structures have been used for improving the sensor performance ${ }^{27,28}$ or developing microbatteries ${ }^{29,30}$. Recently, pyrolytic carbon string resonators have been successfully fabricated with a simple and fast process ${ }^{31,32}$. The optimized micromechanical string resonators display excellent resonant behavior, which has been suitable for applications such as mass sensing and nanomechanical infrared (NAM-IR) spectroscopy $^{33}$. Further optimization of the process resulted in doubly clamped pyrolytic carbon resonators that are electrically conductive, and this property has been explored for the electrodeposition of nanoparticles on suspended structures ${ }^{34}$. Recently, Salazar et al. fabricated suspended pyrolytic carbon nanowires by electromechanical spinning and demonstrated resistive heating that could locally reach temperatures $>2000{ }^{\circ} \mathrm{C}^{35}$.

In this study, we applied electrically conductive pyrolytic carbon resonators as tools for the thermal analysis of nanogram samples of polymers. For this purpose, doubly clamped pre-stressed beam structures served as both micromechanical sensors and resistive elements for integrated heating. First, the electrical resistance and the temperature coefficient of resistance of the pyrolytic carbon were characterized. Next, the resonance frequencies before and after polymer deposition by spray coating were used to estimate the deposited mass. Finally, the resonance frequency and $\mathrm{Q}$ factor of the carbon resonators were measured during a controlled increase of the temperature by resistive heating. The changes in the resonant behavior allowed us to identify the $T_{\mathrm{g}}$ of semicrystalline poly(lactic acid) (PLLA) and $T_{\mathrm{m}}$ of poly (caprolactone) (PCL).

\section{Results and discussion \\ Pyrolytic carbon resonators}

The C-MEMS method was applied for the fabrication of doubly clamped pyrolytic carbon resonators following a process similar to that previously described ${ }^{34}$. The final design of the devices is shown in Fig. 1a. The negative photoresist SU-8 was patterned by standard UV photolithography to define resonators and contact electrodes on a SiN insulation layer. This step was followed by release of the doubly clamped SU-8 beams using isotropic etching and pyrolysis. Finally, Au electrode contacts were deposited by e-beam metal evaporation through a shadow mask. Figure $1 \mathrm{~b}$ shows an SEM image (inset) and the first mode resonance frequency peak of a pyrolytic carbon resonator with a nominal length of $400 \mu \mathrm{m}$, a nominal width of $30 \mu \mathrm{m}$ and a thickness of $0.6 \mu \mathrm{m}$. The resonance frequencies of the pyrolytic carbon resonator were $233 \pm$ $4 \mathrm{kHz}$ for the first mode and $550 \pm 7 \mathrm{kHz}$ for the second mode, which are similar to the values previously reported for devices fabricated under the same pyrolysis conditions $^{34}$. For ideal string-like resonators, the ratio between the frequencies of the second and first modes is 2, whereas for completely stress-free doubly clamped beams, it is $2.76^{36}$. In this study, this ratio was 2.36 , which means that the resonators were not perfect strings but merely pre-stressed doubly clamped beams. Nevertheless, the fabricated pyrolytic carbon resonators should be suitable for thermal analysis of polymers.

\section{Electrical resistance}

The ultimate goal of this study was to apply pyrolytic carbon resonators with integrated temperature control as tools for thermal analysis. To induce resistive heating of the suspended structures by an applied current, the electrical resistance of the resonators had to be in the range of $\mathrm{k} \Omega$. A larger resistance would require high voltages to dissipate the high power, whereas a lower resistance would require high currents to obtain sufficient resistive heating to increase the temperature. The pyrolysis temperature was previously identified as the most important parameter influencing the resistivity of pyrolytic carbon ${ }^{37}$. To adjust the resistance, pyrolytic carbon resonators with pyrolysis temperatures of $700{ }^{\circ} \mathrm{C}, 900{ }^{\circ} \mathrm{C}$, and $1100^{\circ} \mathrm{C}$ were fabricated. The electrical resistances of the pyrolytic carbon resonators were measured using a probe station (Fig. 2a). Figures $2 \mathrm{~b}$, c show the currentvoltage (I-V) curves and the calculated resistance of the pyrolytic carbon resonators with a nominal length of $400 \mu \mathrm{m}$ and a nominal width of $30 \mu \mathrm{m}$ obtained at $900{ }^{\circ} \mathrm{C}$. 

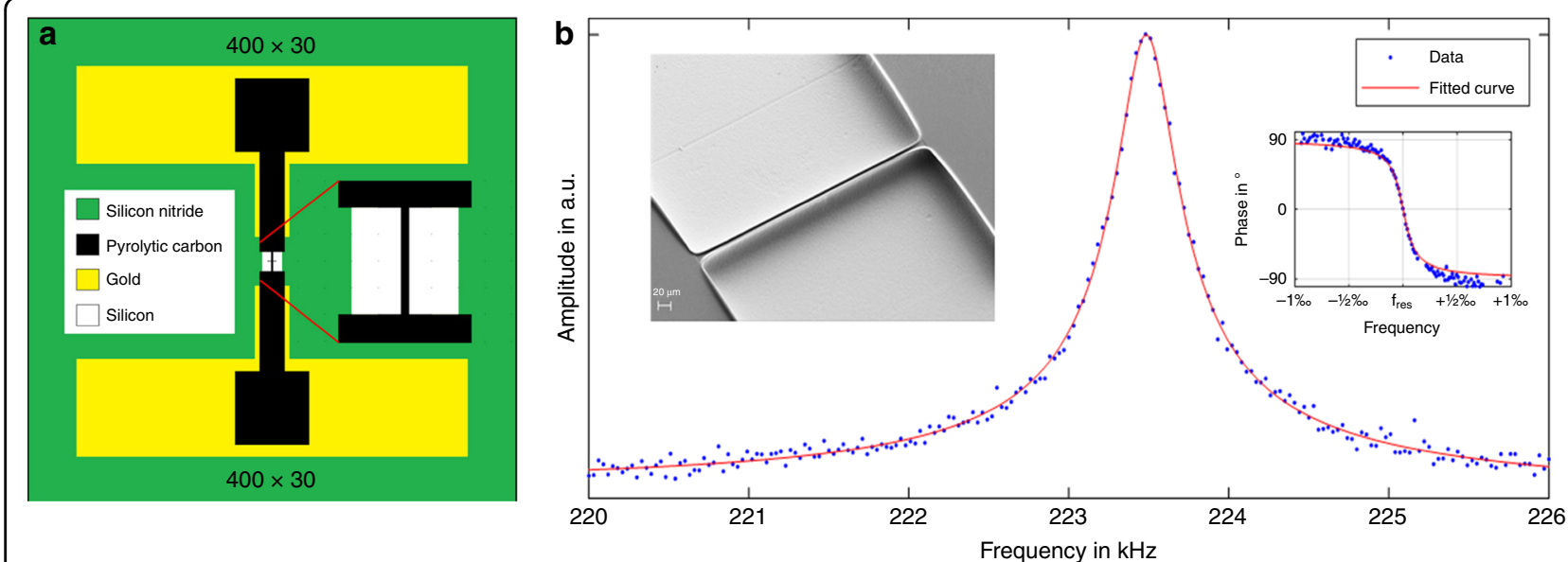

Fig. 1 Pyrolytic carbon resonator. a Design of the doubly clamped pyrolytic carbon resonator; b SEM image (inset) and resonance frequency peak of the first mode

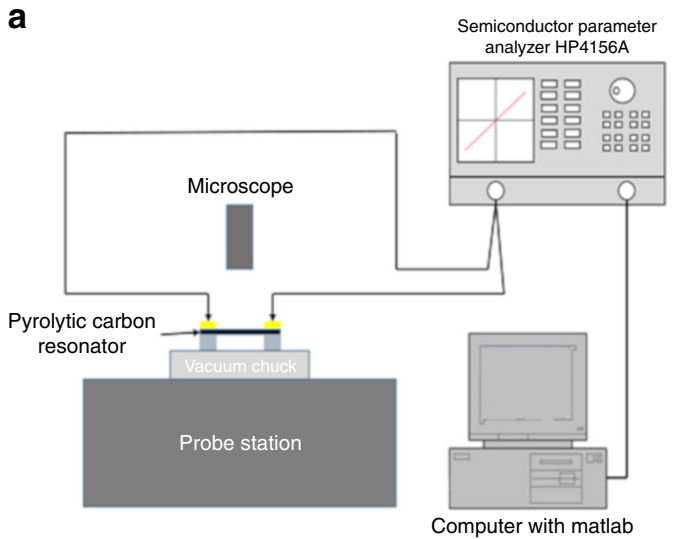

C

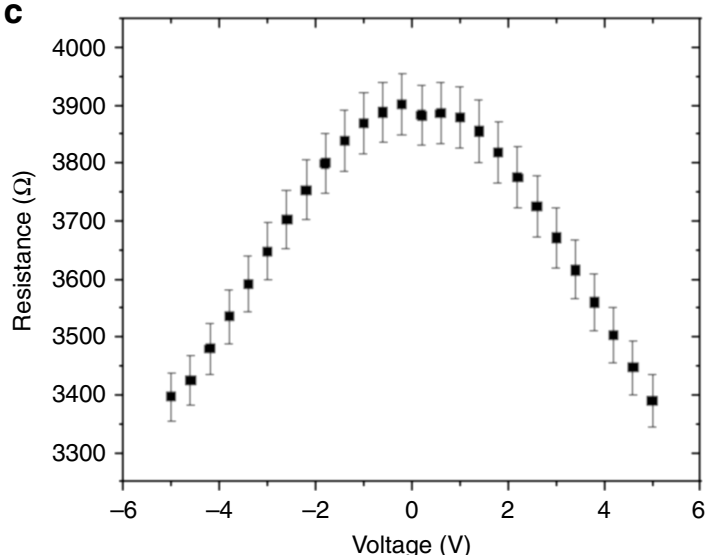

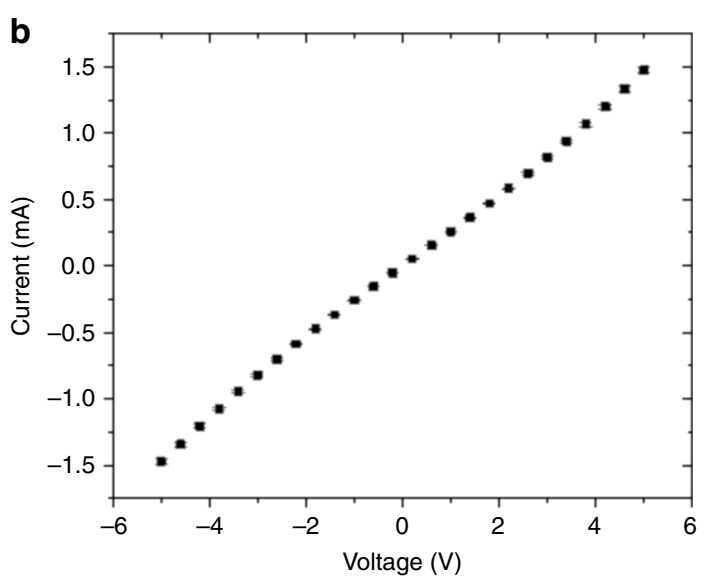

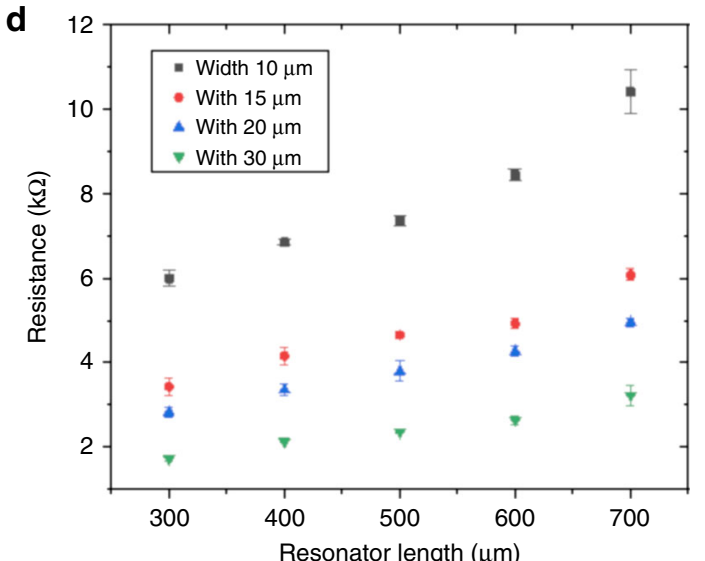

Fig. 2 Electrical characterization of pyrolytic carbon resonators. a probe station setup; $\mathbf{b} \mid-\vee$ curve and $\mathbf{c}$ calculated electrical resistance of pyrolytic carbon resonators with a length of $400 \mu \mathrm{m}$ and a width of $30 \mu \mathrm{m}$ pyrolysed at $900^{\circ} \mathrm{C}$; $\mathbf{d}$ electrical resistances of pyrolytic carbon resonators with various dimensions pyrolysed at $900^{\circ} \mathrm{C}$ 
The results show that the electrical resistance of the resonators displayed an ohmic behavior for small applied potentials in the range of $-2 \mathrm{~V}$ to $2 \mathrm{~V}$. The resistance was calculated as the average value between $-0.5 \mathrm{~V}$ and $0.5 \mathrm{~V}$, giving a value of $3.9 \pm 0.2 \mathrm{k} \Omega$. For larger potentials, a decrease in the resistance was observed (Fig. 2c). This decrease was attributed to the resistive heating of the resonators due to the increasing current. Several resonator designs with different lengths and widths were fabricated for comparison. The electrical resistances of the pyrolytic carbon resonators with different lengths and widths were measured, and the results are shown in Fig. 2d. The results show a linear increase in the resistance with increasing length of the resonators, which confirmed the ohmic behavior of the pyrolytic carbon resistors. With the resistance and the dimensions of the resonators, the resistivity of pyrolytic carbon obtained at a pyrolysis temperature of $900^{\circ} \mathrm{C}$ was calculated to be $16.2 \pm 0.1 \times 10^{-3} \Omega \cdot \mathrm{cm}$, which is comparable with values reported in another study ${ }^{37}$. At the lower pyrolysis temperature of $700{ }^{\circ} \mathrm{C}$, the resistances were three orders of magnitude higher [Supporting Information $\mathrm{S} 1]$; therefore, these devices were not used in further experiments. For resonators pyrolysed at $1100^{\circ} \mathrm{C}$, the resistances were lower [Supporting Information S1]. However, for these structures, buckling due to compressive stress was observed ${ }^{32}$. In conclusion, the resonators fabricated at $900^{\circ} \mathrm{C}$ had resistances in the $\mathrm{k} \Omega$ range and were deemed suitable for resistive heating. For all further experiments, resonators with a nominal length of $400 \mu \mathrm{m}$ and a nominal width of $30 \mu \mathrm{m}$ were used.

\section{Resistive heating of pyrolytic carbon resonators}

To determine the actual temperature of the pyrolytic carbon during resistive heating, the temperature coefficient of resistance (TCR) of pyrolytic carbon had to be measured. The TCR defines the change in resistance as a function of temperature and is calculated as:

$$
\mathrm{TCR}=\frac{R_{1}-R_{0}}{R_{0}\left(T_{1}-T_{0}\right)}
$$

where $R_{0}$ is the resistance at room temperature, $R_{1}$ is the resistance at operating temperature, and $T_{0}$ and $T_{1}$ are room temperature and the operating temperature, respectively.

To determine the TCR of pyrolytic carbon, a Peltier element was used as the external heat source. The pyrolytic carbon resonators were heated up and cooled down for 3 cycles while the resistance was measured. Figure 3a shows that the resistance of the pyrolytic carbon resonators decreased with increasing temperature and that this behavior was repeatable during temperature cycling. The TCR is calculated as the average slope of the resistance value $(\Delta R /$
$\Delta \mathrm{T})$ in the linear interval. Based on Eq. (1), the TCR of pyrolytic carbon was calculated as $-0.0017 \pm 0.0003 \mathrm{ppm} / \mathrm{K}$ $(n=6)$. This negative TCR value of pyrolytic carbon is similar to the negative TCR values reported for graphite $(-0.0005 \mathrm{ppm} / \mathrm{K})^{38}$. A similar decrease in the resistance was observed under external heating of the string resonators based on the readout laser of a vibrometer operated at high power, confirming the negative TCR value for pyrolytic carbon [Supporting Information S2].

For the evaluation of integrated resistive heating, the pyrolytic carbon resonators were mounted on a printed circuit board (PCB), and a current was applied through the suspended structures under high vacuum (Fig. 3b, c). For initial temperature calibration, the actual temperature of the resonators was estimated using the TCR of pyrolytic carbon determined above. The voltage drop across the resonator was measured when applying the current, and the resistance of the carbon resonator was obtained using Ohm's law. With the room temperature $T_{0}$ and the calculated resistances $R_{0}$ and $R_{1}$, Eq. (1) was applied to determine the actual temperature $T_{1}$ of the pyrolytic carbon resonators. Figure $3 \mathrm{~d}$ shows the measured change in resistance as a function of the applied current and the resulting temperature calibration obtained using the TCR of pyrolytic carbon. When the applied current was increased, the resistance of the pyrolytic carbon resonators linearly decreased. As a consequence, the calculated temperature of the resonators increased with a linear dependence on the current, confirming resistive heating. This temperature calibration was used to determine the temperature of the polymer-coated resonators in MTA. Here, it is important to note that the temperature was approximated as an average value for the resonator. The effective temperature profile along the length of the doubly clamped beams should display a maximum value in the center and a lower temperature at the clamped ends.

\section{Deposition of polymer samples}

Polymer samples were deposited onto pyrolytic carbon resonators using spray coating. Figure 4a shows an SEM image after successful deposition of PLLA on the surface of a carbon resonator. The polymer film was uniform on the top surface of the carbon resonator.

Experiments with different numbers of spray passes and different spray speeds were performed to compare the masses of the polymer added to the resonators. For each condition, the resonance frequency of the resonators was measured before and after the deposition to estimate the added mass based on the following equation ${ }^{36}$ :

$$
\Delta m=m_{0}\left(\left(\frac{f_{0}}{f_{0}-\Delta f}\right)^{2}-1\right)
$$



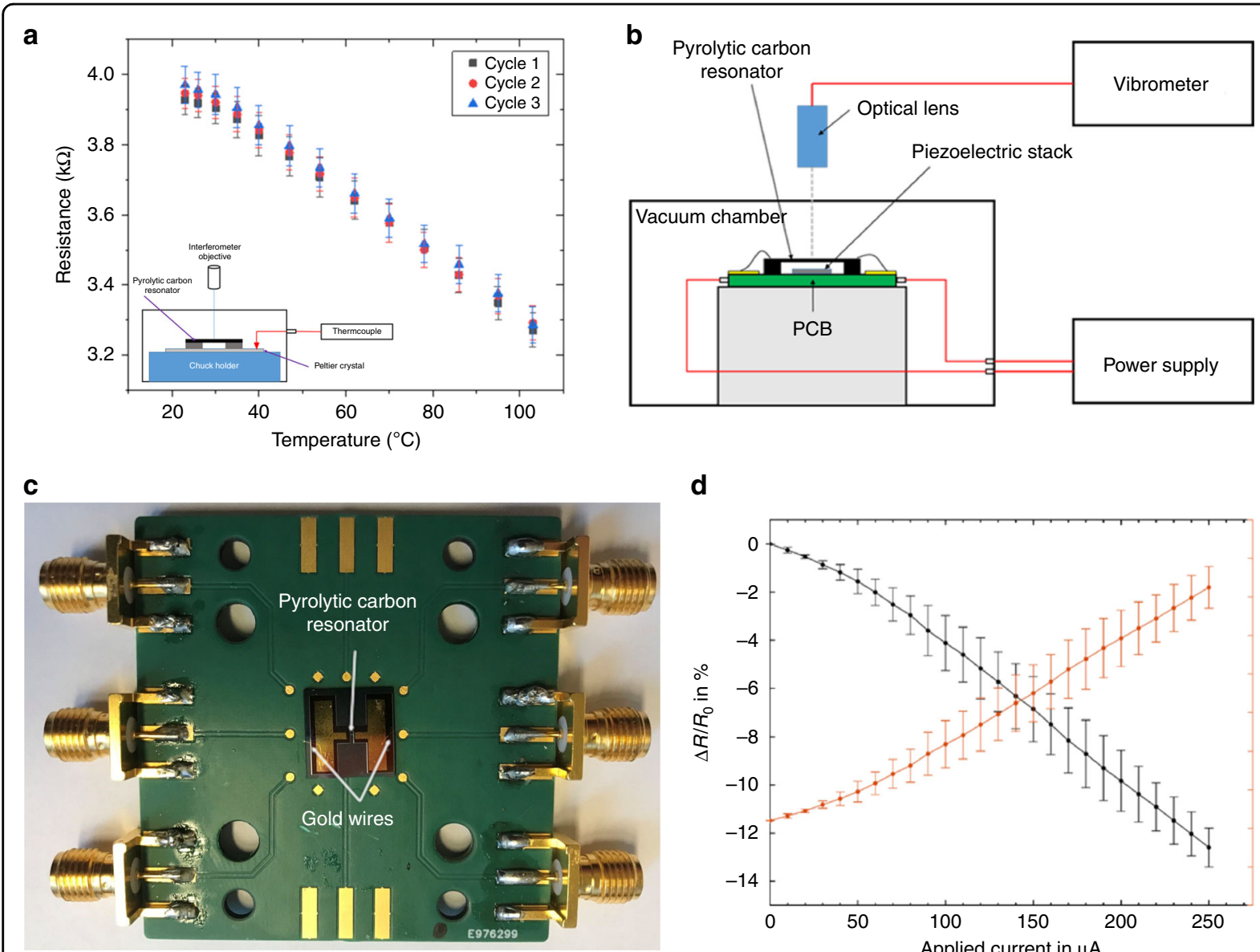

d

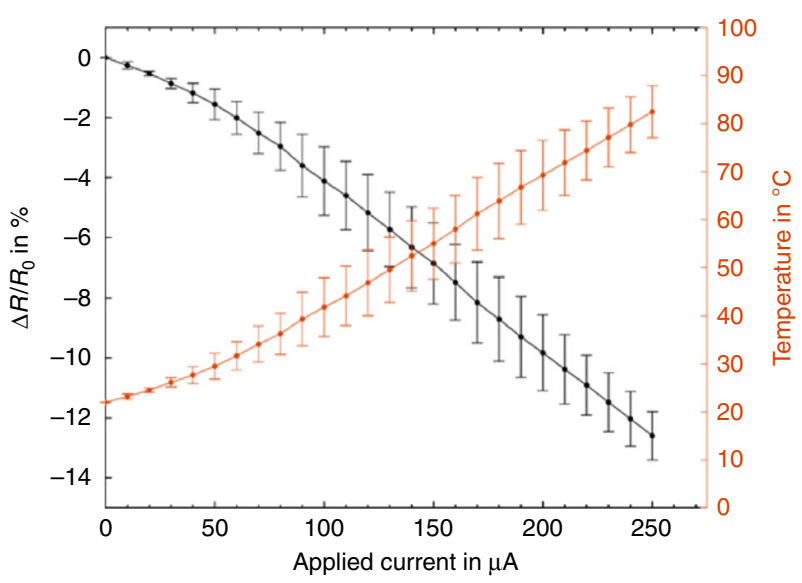

Fig. 3 Integrated resistive heating characterization. a Resistance of pyrolytic carbon resonators as a function of temperature externally controlled by a Peltier element (inset); $\mathbf{b}$ experimental setup for integrated resistive heating of pyrolytic carbon resonators; $\mathbf{c}$ device mounted on PCB; $\mathbf{d}$ measured change in resistance due to resistive heating, and calculated temperature of pyrolytic carbon resonators

where $\Delta m$ is the change in mass, $\Delta f$ is the change in the resonance frequency, and $m_{o}$ and $f_{o}$ are the mass and resonance frequency of the resonator before deposition, respectively. Equation (2) is based on the approximation that the effective stiffness of the resonator is constant before and after deposition and that the deposited mass is distributed evenly over the length of the resonator. To calculate the added mass using Eq. (2), the density of the pyrolytic carbon had to be estimated. With the density measurement, the density of pyrolytic carbon was calculated as $1.36 \pm 0.18 \mathrm{~g} / \mathrm{cm}^{3}$, which is similar to values reported in another study $\left(1.52 \pm 0.06 \mathrm{~g} / \mathrm{cm}^{3}\right)^{39}$. The slight difference between the two values can be explained by the difference in pyrolysis process parameters. Figure $4 \mathrm{~b}$, c summarize the change in resonance frequency and the added mass for different numbers of spray passes and different spray speeds. For all the samples, $<5 \mathrm{ng}$ of PLLA was deposited. The results show linear decreases in the resonance frequency shift and the added mass when the spray speed was increased for both one and two spray passes. In both cases, less polymer was deposited at higher spray speeds, as would be expected. Meanwhile, when the number of spray passes was increased from one to two, more polymer was successfully deposited on the beams, leading to larger resonance frequency shifts, as well as more added mass. With increasing thickness of the deposited polymer layer, the assumption of constant resonator stiffness will increasingly lead to underestimation of the added mass.

\section{Micromechanical thermal analysis of polymers}

The micromechanical thermal analysis of polymers in this study is based on resonance frequency measurements of pyrolytic carbon resonators during temperature ramping. Therefore, first, the resonant behavior of uncoated (blank) pyrolytic carbon resonators upon heating was investigated. The temperature of a resonator was increased by resistive heating while the resonance frequency and $Q$ factor of the carbon resonator were recorded. Figure $5 \mathrm{a}-\mathrm{c}$ show that when the temperature 


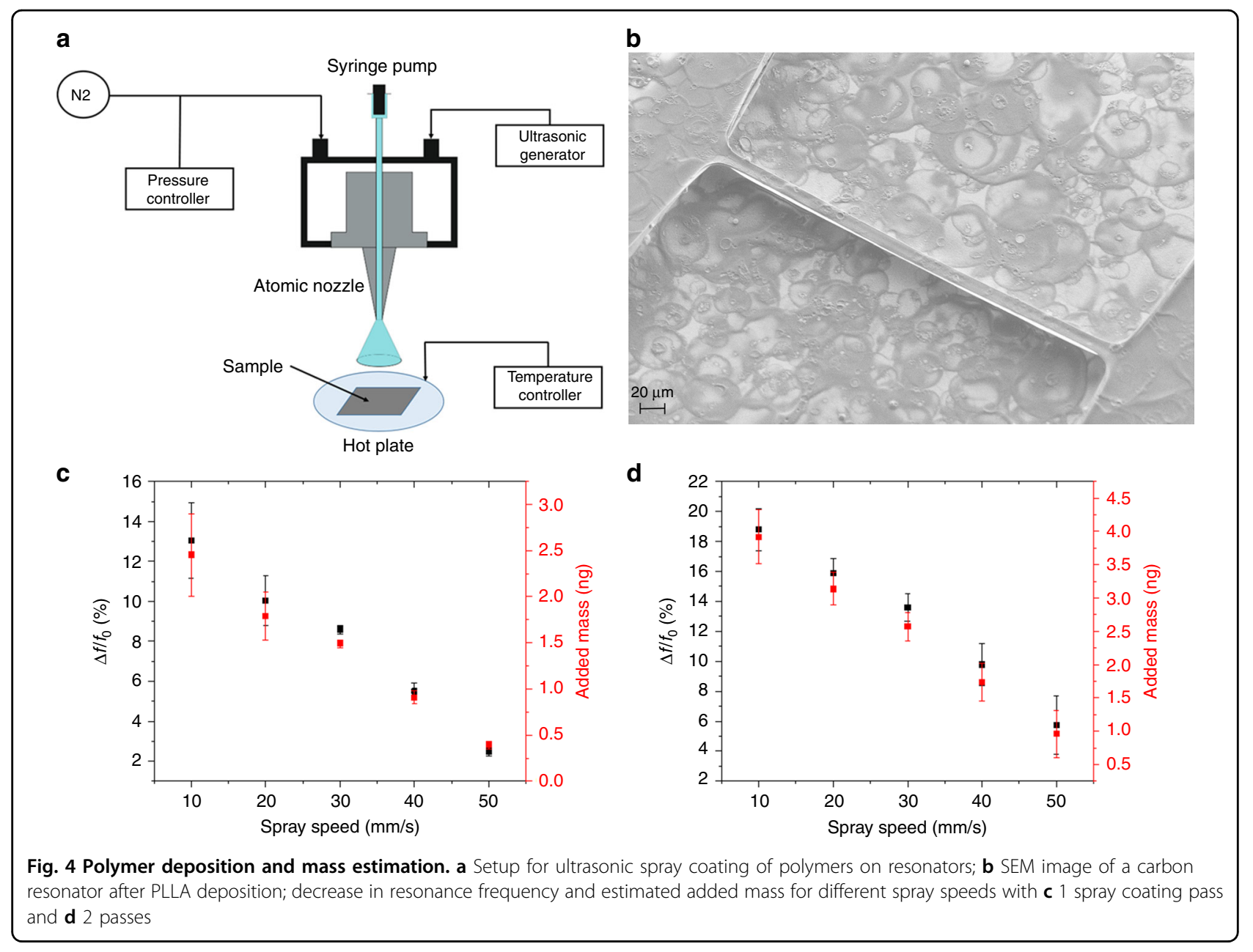

was increased, the resonance frequency increased. The resistive heating is limited to the pyrolytic carbon resonators. Therefore, this observation indicates an increase in the tensile stress in the doubly clamped beam due to the negative coefficient of thermal expansion (CTE) of the pyrolytic carbon. For temperatures $<200{ }^{\circ} \mathrm{C}$, negative values of CTE have frequently been reported for other carbon materials such as graphite or pyrolytic carbon deposited by chemical vapor deposition ${ }^{40,41}$. A similar increase in the resonance frequency was observed under external local heating of the string resonators based on the readout laser of a vibrometer operated at high power [Supporting Information S2], confirming the negative CTE for pyrolytic carbon. In terms of the $\mathrm{Q}$ factor, the value was stable when the temperature increased, indicating a negligible change in resonator damping.

For the micromechanical thermal analysis, PLLA was spray coated on pyrolytic carbon resonators (2 passes, $30 \mathrm{~mm} / \mathrm{s}$ ). The polymer-coated carbon resonators were heated by resistive heating under the same conditions as the blank resonators. Figure $5 \mathrm{~d}-\mathrm{f}$ show the thermal analysis of PLLA using three different pyrolytic carbon resonators. Two different temperature regimes were identified. In the first regime, a linear decrease in the resonance frequency was observed until the resonators reached a temperature of $\sim 60^{\circ} \mathrm{C}$. After this point, in the second regime, the resonance frequency linearly increased. A similar behavior was observed for the Q factor of the resonators. The initial decrease in the resonance frequency was attributed to the thermal expansion of the PLLA with a positive CTE $E_{\text {PLLA }}=58.4 \mathrm{ppm} / \mathrm{K}^{8}$, in contrast to the negative CTE of the pyrolytic carbon. The change in slope at approximately $60^{\circ} \mathrm{C}$ is related to the glass transition temperature $T_{\mathrm{g}}$ of PLLA. During the glassrubber phase transition, the Young's modulus of PLLA considerably decreases ${ }^{42}$. Therefore, the mechanical properties of the resonator above $T_{\mathrm{g}}$ are dominated by the properties of the pyrolytic carbon. Hence, after passing $T_{\mathrm{g}}$, the resonance frequency increased as a function of temperature, as was observed for the blank carbon resonators. Based on the minimum value of the resonance frequency, $T_{\mathrm{g}, \text { PLLA }}$ was determined to be $61.0 \pm 0.8^{\circ} \mathrm{C}(n=3)$. This temperature was comparable with the $T_{\mathrm{g}, \text { PLLA }}$ of $60.8^{\circ} \mathrm{C}$ measured by $\mathrm{DSC}^{43}$. The gradual decrease and 

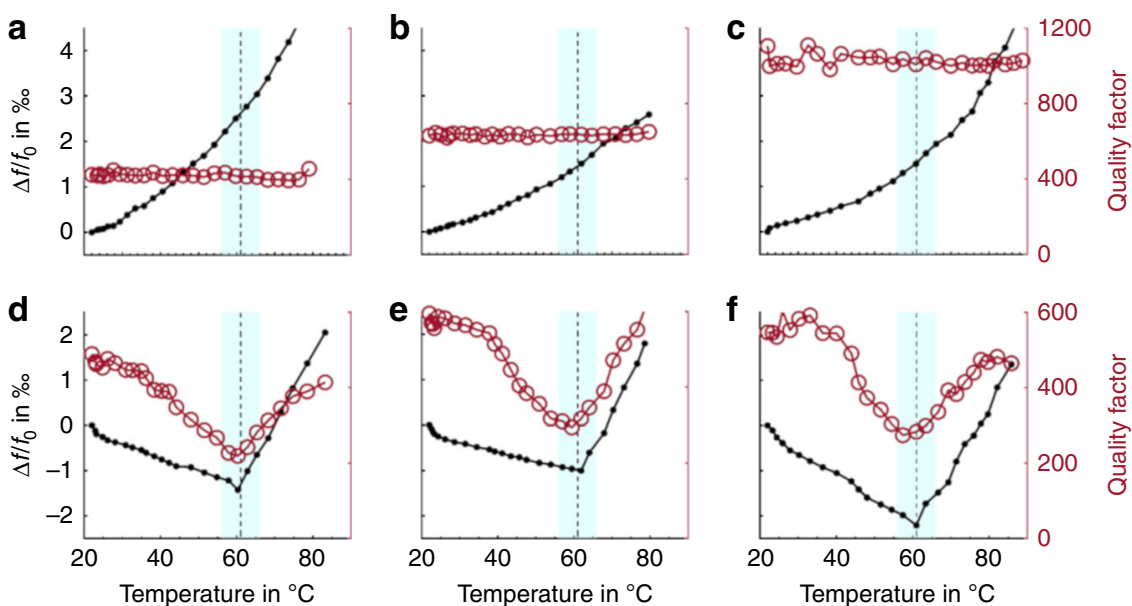

Fig. 5 Micromechanical thermal analysis using pyrolytic carbon resonators. a-c before and $\mathbf{d}-\mathbf{f}$ after poly(lactic acid) coating

subsequent increase in the $\mathrm{Q}$ factor is caused by the gradual increase in molecular movement within the polymer, which results in a maximum of the viscous damping within the polymer at $T_{\mathrm{g}}$. Above this temperature, the mechanical strength of the polymer layer decreases, which reduces the damping contribution of the polymer layer, and the Q factor recovers.

For further investigation of the potential of pyrolytic carbon resonators as a tool for thermal analysis, PCL was deposited on pyrolytic carbon resonators by spray coating using the same parameters as for PLLA. Figure 6 shows the thermal analysis of PCL using resistive heating of three different pyrolytic carbon resonators. At low temperatures, the resonance frequency of the coated resonators remained relatively stable. However, above $60{ }^{\circ} \mathrm{C}$, a step-shaped increase in the resonance frequency was observed. This change in resonant behavior was attributed to the melting of PCL at the melting temperature $T_{\mathrm{m}, \mathrm{PCL}}$. At $T_{\mathrm{m}}$, a polymer changes from a semicrystalline state to an amorphous state, in principle losing all pre-orientation of the polymer chains. This phase change might result in a loss of structural integrity of the polymer and less damping of the resonator, leading to recovery of the $\mathrm{Q}$ factor. Furthermore, reorganization of mass on the surface of the resonators, e.g., due to polymer reflow, can lead to changes in the effective resonator mass, resulting in a sudden change in the resonance frequency ${ }^{9}$. Based on the change in slope in the frequency measurements, the melting temperature of PCL was determined to be $T_{\mathrm{m}, \mathrm{PCL}}$ $=60.0 \pm 1.1^{\circ} \mathrm{C}$, which is similar to the melting temperature of PCL reported in the literature $\left(57.8 \pm 4{ }^{\circ} \mathrm{C}\right)^{44}$.

\section{Conclusion}

In this study, electrically conductive pyrolytic carbon resonators were developed and established as a tool for micromechanical thermal analysis. With a simple fabrication process, the pyrolytic carbon resonators show good resonant behavior with a resonance frequency of $233 \mathrm{kHz}$ and $Q$ factors between 600 and 1000 in vacuum. The carbon resonators are suitable for resistive heating using currents in the range of $\mu \mathrm{A}$. The resistive heating allows for internal temperature control of the carbon resonators and the possibility to perform temperature ramping during resonance frequency measurements. Furthermore, the temperature coefficient of resistance of pyrolytic carbon is negative and estimated to be $-0.0017 \pm 0.0003 \mathrm{ppm} / \mathrm{K}$. After temperature calibration, the sensors were used as a tool for thermal analysis of the glass transition temperature $\left(T_{\mathrm{g}}\right)$ of PLLA and melting temperature $\left(T_{\mathrm{m}}\right)$ of PCL. Compared to heating with an external Peltier element, the integrated resistive heating allows measurements immediately after increasing the temperature due to the low thermal mass of the system. The results of the micromechanical thermal analysis give $T_{\mathrm{g}, \text { PLLA }}=61.0 \pm 0.8^{\circ} \mathrm{C}$ and $T_{\mathrm{m}, \mathrm{PCL}}=60.0 \pm 1.1^{\circ} \mathrm{C}$, which are in excellent agreement with the literature. Furthermore, our results indicate a negative coefficient of thermal expansion (CTE) for pyrolytic carbon. In the future, we will explore whether the temperature range for thermal analysis with pyrolytic carbon resonators can be further increased. The results show the promising potential of conductive pyrolytic carbon resonators for micromechanical thermal analysis.

\section{Materials and methods}

\section{Pyrolytic carbon resonator fabrication}

The fabrication process of pyrolytic carbon resonators used in this study was previously described in detail ${ }^{34}$. Briefly, pyrolytic carbon resonators were fabricated using silicon nitride dry etching, SU-8 photolithography, isotropic silicon etching, pyrolysis and e-beam metal 

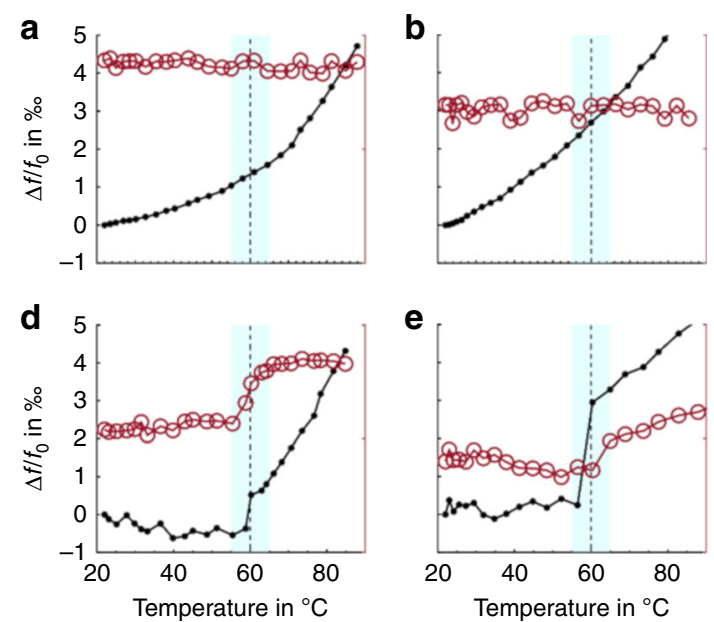
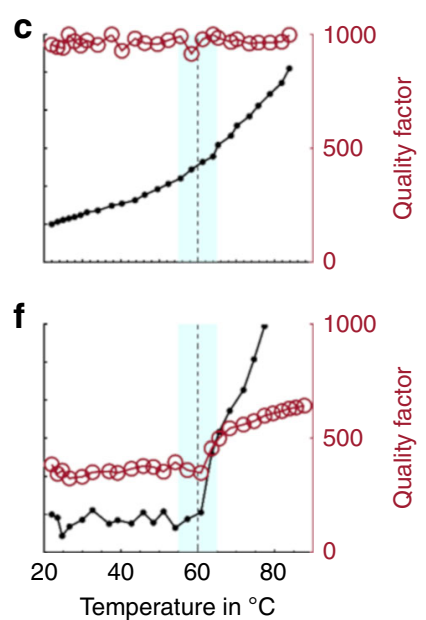

Fig. 6 Micromechanical thermal analysis using pyrolytic carbon resonators. $\mathbf{a}-\mathbf{c}$ before and $\mathbf{d}-\mathbf{f}$ after poly(caprolactone) coating

evaporation. However, in this work, the design of the resonators (Fig. 1a) was modified, and the thickness of the resonators was reduced to $0.6 \mu \mathrm{m}$. Pyrolytic carbon resonators with different lengths and widths were prepared for characterization of the electrical resistance.

\section{Electrical resistance measurement}

The electrical resistances of the carbon resonators were measured by a probe station and a Semiconductor Parameter Analyzer HP4156A (Fig. 1b). The applied voltage was varied from $-5 \mathrm{~V}$ to $5 \mathrm{~V}$ while the current was measured. The Semiconductor Parameter Analyzer HP4156A was controlled by MATLAB software to obtain the $\mathrm{I}-\mathrm{V}$ curves and the resistance values between the two terminals.

\section{Polymer preparation and spray coating process}

To investigate the potential of the pyrolytic carbon resonators as a tool for thermal analysis, two polymers were characterized, and $0.5 \mathrm{wt} . \%$ solutions of poly(L-lactic acid) (PLLA) ( $M_{\mathrm{w}} 204,800 \mathrm{~g} / \mathrm{mol}$, NatureWorks PLA polymer 2003D) and poly(caprolactone) (PCL) $\left(M_{\mathrm{w}}\right.$ $80,000 \mathrm{~g} / \mathrm{mol}$, Sigma Aldrich) with dichloromethane (DCM, Sigma Aldrich) as a solvent were prepared. The polymer solutions were spray coated on one side of the pyrolytic carbon resonators with an Exacta Coat Ultrasonic Spray System (Sonotek, USA) in a way similar to that previously described (Fig. 4a) ${ }^{8}$. The tip of the ultrasonic atomizer nozzle (Accumist, Sonotek, USA) was actuated at a frequency of $120 \mathrm{kHz}$ with a generator power of $1.3 \mathrm{~W}$, compressed nitrogen for shaping air at $0.04 \mathrm{kPa}$ and a solution flow rate of $0.1 \mathrm{ml} / \mathrm{min}$. In this work, the distance from the nozzle tip to the substrate was fixed at $4 \mathrm{~cm}$, and the temperature was kept constant at room temperature. The translational movement speed of the spraying nozzle $(10-50 \mathrm{~mm} / \mathrm{s})$ and the number of times the nozzle passed over the substrates ( 1 or 2 passes) were changed to deposit different masses of polymer on the resonators.

\section{Density measurement}

For the estimation of the added mass of spray-coated polymers, the density and thickness of the pyrolytic carbon string resonator material had to be determined. For this purpose, unpatterned SU-8 photoresist films were processed on 4 inch $\mathrm{Si}$ wafers using parameters identical to those for the fabrication of the string resonators. The samples were weighed on a microbalance before spincoating and after pyrolysis of the polymer films to obtain the weight of the pyrolytic carbon. The carbon layer was mechanically removed at several points across the wafer, and the film thickness was determined using a Dektak 8 stylus profilometer.

\section{Micromechanical thermal analysis}

The thermal analysis of the polymers was based on resonance frequency measurements of the pyrolytic carbon resonators. The resonance frequency was tracked using a commercial MSA-500 laser Doppler vibrometer from Polytec and an HF2LI lock-in amplifier from Zurich Instruments as previously described ${ }^{32}$. For temperature calibration, external heating was used, and the chip with the pyrolytic carbon resonators was placed on top of a Peltier element. The temperature was controlled by a thermocouple connected to the hot side of the Peltier element. The temperature was gradually increased, and the electrical resistance of the pyrolytic carbon resonators was recorded $10 \mathrm{~min}$ after changing the set value to allow for stabilization of the temperature. These measurements were used as calibration curves to determine the 
temperatures achieved by integrated resistive heating. For resistive heating, the pyrolytic carbon resonators were wire bonded to a printed circuit board (PCB), and a current was applied through the resonators (Fig. 3b, c). For thermal analysis, the applied current was stepwise increased, which resulted in an increase in the temperature of the resonators due to resistive heating of the carbon. Resistance measurements were performed immediately after setting the new temperature value. During the heating process, the resonance frequencies of the carbon resonators (blank and polymer coated) were monitored.

\section{Acknowledgements}

The authors thank Chiara Mazzoni for the spray coating process support. The research is funded by the Danish National Research Foundation (DNRF122) and Villum Fonden (Grant No. 9301) Center for Intelligent Drug Delivery and Sensing Using Microcontainers and Nanomechanics (IDUN). This work was performed in part at cleanroom facilities of DTU Nanolab, the National Center for Microfabrication and Nanofabrication at the Technical University of Denmark.

\section{Author details}

'DNRF and Villum Fonden Center for Intelligent Drug Delivery and Sensing Using Microcontainers and Nanomechanics, IDUN, Technical University of Denmark, 2800 Kgs Lyngby, Denmark. ${ }^{2}$ DTU Nanolab, Technical University of Denmark, 2800 Kgs Lyngby, Denmark. ${ }^{3}$ DTU Health Tech, Technical University of Denmark, 2800 Kgs Lyngby, Denmark. ${ }^{4}$ Advanced NEMS Laboratory, Ecole Polytechnique Fédérale de Lausanne, 1015 Lausanne, Switzerland

\section{Author's contributions}

L.Q.N., S.S.K., P.E.M., and A.B. had the idea and planned the experimental study. L.Q.N. and S.S.K. designed the devices and the fabrication process. L.Q.N. carried out cleanroom fabrication of the carbon resonators. L.Q.N., T.L., and L.G. V. planned and executed the measurements of the electrical resistance. L.Q.N. and P.E.M. prepared the experimental setup for resistive heating and micromechanical thermal analysis. S.B.G. implemented polymer deposition on the resonators. L.Q.N. and P.E.M. performed MTA measurements and the data treatment. L.Q.N. and S.S.K. wrote the paper. All of the authors commented on the paper.

\section{Conflict of interest}

The authors declare that they have no conflict of interest.

Supplementary information accompanies this paper at https://doi.org/ 10.1038/s41378-019-0094-x.

Received: 19 March 2019 Revised: 12 June 2019 Accepted: 22 July 2019 Published online: 21 October 2019

\section{References}

1. Chan Sze On, D. R. H. An overview of thermal analysis of polymers. J. Sci. Technol. Dev. 3, 54 (2017).

2. Wunderlich, B. Methodology of interpreting thermal analysis of polymers. J. Therm. Anal. Calorim. 106, 85-91 (2011).

3. Attia, A. K. Mohamed Ibrahim, M. \& Nabi El-Ries, M. A. Thermal analysis of some antidiabetic pharmaceutical compounds. Adv. Pharm. Bull. 3, 419-424 (2013).

4. Magoshi, J., Becker, M. A., Han, Z. \& Nakamura, S. Thermal properties of seed proteins. J. Therm. Anal. Calorim. 70, 833-839 (2002).

5. Berger, R. et al. Micromechanical thermogravimetry. Chem. Phys. Lett. 294, 363-369 (1998)

6. Mansfield, E., Kar, A., Quinn, T. P. \& Hooker, S. A. Quartz crystal microbalances for microscale thermogravimetric analysis. Anal. Chem. 82, 9977-9982 (2010).
7. Berger, R., Gerber, C., Gimzewski, J. K., Meyer, E. \& Guntherodt, H. J. Thermal analysis using a micromechanical calorimeter. Appl. Phys. Lett. 69, 40-42 (1996).

8. Bose, S. et al. Micromechanical string resonators: analytical tool for thermal characterization of polymers. A C. S Macro Lett. 3, 55-58 (2014).

9. Karl, M. et al. Ultra sensitive micro string resonators for solid state thermomechanical analysis of small and large molecules. J. Am. Chem. Soc. 140, 17522-17531 (2018).

10. Li, B.-B. et al. On chip, high-sensitivity thermal sensor based on high-Q polydimethylsiloxane-coated microresonator. Appl. Phys. Lett. 96, 251109 (2010).

11. He, L., Xiao, Y.-F., Zhu, J., Ozdemir, S. K. \& Lan, Y. Oscillatory thermal dynamics in high-Q PDMS-coated silica toroidal microresonators. Opt. Express 17, 9571-9581 (2009)

12. Jung, N., Seo, H., Lee, D., Ryu, C. Y. \& Jeon, S. Nanomechanical Thermal Analysis of the Glass Transition of Polystyrene Using Silicon Cantilevers. Macromolecules 41, 6873-6875 (2008).

13. Manzoor Bukhari, S. A., Khan, M. F., Goswami, A., McGee, R. \& Thundat, T. Thermomechanical analysis of picograms of polymers using a suspended microchannel cantilever. RSC Adv. 7, 8415-8420 (2017).

14. Soccio, M. et al. Thermomechanical response of a semicrystalline polymer in the vicinity of the melting by using microcantilever technology. Appl. Phys. Lett. 104, 251904 (2014)

15. Ahumada, O. et al. Sensitive thermal transitions of nanoscale polymer samples using the bimetallic effect: Application to ultra-thin polythiophene. Rev. Sci. Instrum. 84, 053904 (2013).

16. Alves, G. M. A., Bose-Goswami, S., Mansano, R. D. \& Boisen, A. Temperature Modulated Nanomechanical Thermal. Anal. I E E E Sens. J. 18, 4001-4007 (2018).

17. Yim, C. et al. Nanomechanical Thermal Analysis of Indium Films Using Silicon Microcantilevers. Jpn. J. Appl. Phys. 51, $08 \mathrm{KBO7}$ (2012).

18. Zampetti, E. et al. Exploitation of an integrated microheater on QCM sensor in particulate matter measurements. Sens. Actuators, A: Phys. 264, 205-211 (2017).

19. Jakoby, B., Klinger, F. P. \& Svasek, P. A novel microacoustic viscosity sensor providing integrated sample temperature control. Sens. Actuators, A: Phys. 123-124, 274-280 (2005).

20. Chung, G. S. \& Jeong, J. M. Fabrication of micro heaters on polycrystalline 3CSiC suspended membranes for gas sensors and their characteristics. Microelectron. Eng. 87, 2348-2352 (2010).

21. Greve, A. et al. Micro-calorimetric sensor for vapor phase explosive detection with optimized heat profile. Microelectron. Eng. 87, 696-698 (2010).

22. Wang, C. L. \& Madou, M. From MEMS to NEMS with carbon. Biosens. Bioelectron. 20, 2181-2187 (2005)

23. Martinez-Duarte, R. SU-8 Photolithography as a Toolbox for Carbon MEMS Micromachines 5, 766-782 (2014).

24. Ranganathan, S., McCreery, R., Majii, S. M. \& Madou, M. Photoresist-derived carbon for microelectromechanical systems and electrochemical applications. J. Electrochem. Soc. 147, 277-282 (2000).

25. Fischer, D. J., Vandaveer, W. R. IV, Grigsby, R. J. \& Lunte, S. M. Pyrolyzed photoresist carbon electrodes for microchip electrophoresis with dualelectrode amperometric detection. Electroanalysis 17, 1153-1159 (2005).

26. Hirabayashi, M., Mehta, B., Vahidi, N. W., Khosla, A. \& Kassegne, S. Functionalization and characterization of pyrolyzed polymer based carbon microstructures for bionanoelectronics platforms. J. Micromech. Microeng. 23, 115001 (2013).

27. $\mathrm{Xu}, \mathrm{H}$. et al. Carbon post-microarrays for glucose sensors. Biosens. Bioelectron. 23, 1637-1644 (2008).

28. Kassegne, S., Wondimu, B., Majzoub, M. \& Shin, J. High-efficiency microarray of 3-D carbon MEMS electrodes for pathogen detection systems. Proc. Spie - Int. Soc. Opt. Eng. 7266, 726615 (2008).

29. Wang, C., Jia, G., Taherabadi, L. H. \& Madou, M. J. A novel method for the fabrication of high-aspect ratio C-MEMS structures. J. Micro. Syst. 14, 348-358 (2005).

30. Wang, C. et al. C-MEMS for the manufacture of 3D microbatteries. Electrochem. Solid-State Lett. 7, A435-A438 (2004).

31. Kurek, Maksymilian et al. Sensors 16, 1097 (2016).

32. Quang, LongNguyen, Larsen, PeterEmil, Boisen, Anja \& Keller, StephanSylvest Tailoring stress in pyrolytic carbon for fabrication of nanomechanical string resonators. Carbon 133(July), 358-368 (2018).

33. Nguyen, L. Q., Larsen, P. E., Bishnoi, S., Boisen, A. \& Keller, S. S. Fabrication of fully suspended pyrolytic carbon string resonators for characterization of drug nano- and microparticles. Sens. Actuators. 288, 194-203 (2019). 
34. Quang, L. N. et al. Electrochemical pyrolytic carbon resonators for mass sensing on electrodeposited polymers. Micro Nano Eng. 2, 64-69 (2019).

35. Salazar, A., Cardenas-Benitez, B., Pramanick, B., Madou, M. J. \& Martinez-Chapa, S. O. Nanopag fabrication by Joule heating of electromechanically spun suspended carbon nanofibers. Carbon 115, 811-818 (2017).

36. Schmid, S., Villanueva, L. G., \& Roukes, M. L. Fundamentals of nanomechanical resonators. (pp. 1-175). (Springer International Publishing, 2016).

37. Hassan, Y. M. et al. High temperature SU-8 pyrolysis for fabrication of carbon electrodes. J. Anal. Appl. Pyrolysis 125, 91-99 (2017).

38. Giancoli, Douglas C. Physics, 4th edn. (Prentice Hall, 1995).

39. Kurek, M. et al. Nanomechanical pyrolytic carbon resonators: novel fabrication method and characterization of mechanical properties. Sensors 16, 1097 (2016).
40. Mag-isa, A. E., Kim, J.-H. \& Oh, Ch-S. Measurements of the in-plane coefficient of thermal expansion of freestanding single-crystal natural graphite. Mater. Lett. 171, 312-314 (2016).

41. Zhang, D., Li, H., Jia, Y., Guo, L. \& Li, H. Coefficients of thermal expansion of low texture and isotropic pyrocarbon deposited on stationary substrates. Mater. Lett. 68, 68-70 (2012).

42. Sperling, L. H. Introduction to Physical Polymer Science. (John Wiley \& Sons, Inc, Hoboken, 2005)

43. Neumann, I. A., Sydenstricker Flores-Sahagun, T. H. \& Ribeiro, A. M. Biodegradable poly (L-lactic acid) (PLLA) and PLLA-3-arm blend membranes: the use of PLLA-3-arm as a plasticizer. Polym. Test. 60, 84-93 (2017).

44. Ali, S. F. A. Mechanical and thermal properties of promising polymer composites for food packaging applications. lop Conf. Ser. 137, 012035 (2016). 